\title{
Factors Affecting Early Treatment Goals of Sepsis Patients Presenting to the Emergency Department
}

\author{
C. K. Kassyap, Siju V. Abraham, S. Vimal Krishnan, Babu Urumese Palatty, P. C. Rajeev \\ Department of Emergency Medicine, Jubilee Mission Medical College and Research Institute, Thrissur, Kerala, India
}

\section{Abstract}

Background and Objectives: Sepsis is a major cause of emergency medicine admission. It is associated with high mortality and morbidity. Even though sepsis is common in the Indian subcontinent, there is a paucity of data on the management of sepsis in India. The aim was to study the factors affecting early treatment goals. Methods: All clinically suspected sepsis patients consenting to be part of the study were included. The diagnosis of sepsis was made by the treating physician in the emergency department as per the Surviving Sepsis Guidelines criteria. All cases were managed as per institutional treatment protocol. The patients were prospectively followed up and the time taken to achieve the goal-directed sepsis bundle documented and analyzed. Results and Discussion: Of the 75 patients studied, the 3-hour(h) bundles were achieved in $70.7 \%$ of cases and 6-h bundles were achieved in $84 \%$ of cases. Meantime for obtaining blood culture was 107 min and administration of first dose antibiotics was $134 \mathrm{~min}$. Thirty patients failed to achieve the early treatment goals, of which six were under-triaged, seven due to physicians delay in recognizing sepsis, 11 due to logistical delay, and six were due to financial constraints. Conclusion: The sepsis bundle goals were not achieved because of various factors such as under triaging, delay in diagnosis, logistical delay, and financial constraints. Further studies on whether sensitization of medical fraternity about sepsis, implementation of insurance policies for patient care or better point of care diagnostics would aid in achieving the bundles may be evaluated further.

Keywords: Early goal-directed therapy, sepsis, sepsis bundle care, Surviving Sepsis Guidelines Campaign

\section{INTRODUCTION}

Sepsis is a clinical syndrome that complicates severe infection. It is characterized by cardinal signs of inflammation (vasodilatation, leukocytosis, and increased microvascular permeability) occurring in tissue that are remote from the site of infection. It is a major cause of morbidity and mortality and the second leading cause of death worldwide. ${ }^{[1-3]}$

The delay in diagnosis and management of sepsis will cause an increase in mortality and morbidity. Early intervention can prevent the progression of sepsis to septic shock and multiple organ dysfunction syndrome. ${ }^{[4]}$ This study aims to identify those factors which cause delay in the management of sepsis.

\section{Methods}

The study was conducted as a prospective observational study at an Emergency Medicine (EM) Department of a tertiary care hospital in South India. The study protocol was reviewed and

\begin{tabular}{|l|l|}
\hline \multicolumn{3}{c|}{ Access this article online } \\
\hline Quick Response Code: & Website: \\
& www.ijccm.org \\
& \\
\end{tabular}

approved by the Institutional Ethical Committee (Ref no: 75/15/IEC JMMC and RI).

All adults ( $\geq 18$ years of age), with a clinical diagnosis of sepsis presenting to the Department of EM fulfilling the sepsis diagnostic criteria [Table 1] were included if they consented to be part of the study. The cases were managed as per institutional treatment protocol for sepsis. The patients were prospectively followed up and the time taken to achieve the goal-directed sepsis bundles were documented by an independent observer. ${ }^{[5]}$ If the goals were not achieved in the specified time (i.e., 3-h and 6-h, respectively), the reason for the delay was noted by the observer as under triaged, physician delay, logistical delay, or financial constraints.

Address for correspondence: Dr. Siju V. Abraham, Department of Emergency Medicine, Jubilee Mission Medical College and Research Institute, Bishop Alapatt Road, Thrissur - 680 005, Kerala, India. E-mail: sijuvabraham@gmail.com

This is an open access journal, and articles are distributed under the terms of the Creative Commons Attribution-NonCommercial-ShareAlike 4.0 License, which allows others to remix, tweak, and build upon the work non-commercially, as long as appropriate credit is given and the new creations are licensed under the identical terms.

For reprints contact: reprints@medknow.com

How to cite this article: Kassyap CK, Abraham SV, Krishnan SV, Palatty BU, Rajeev PC. Factors affecting early treatment goals of sepsis patients presenting to the emergency department. Indian J Crit Care Med 2018;22:797-800. 


\section{Statistical methods}

Descriptive and inferential statistical analysis have been carried out in the present study. Results on continuous measurements are presented as mean \pm standard deviation, median and minimum-maximum. The International Business Machines Statistical Package for Social Sciences (IBM Corp. Released 2011. IBM SPSS Statistics for Windows, Version 20.0. IBM Corp., Armonk, NY, USA) was used for the analysis of the data. Microsoft Word and Microsoft Excel (2007 version) were used to enter data and generate graphs, tables, and charts.

\section{RESULTS}

In our study, 3-h bundle was achieved in $70.7 \%$ of cases [Table 2] and 6-h bundle was achieved in $84 \%$ of cases [Table 3].

The mean time taken for lactate measurement was $20.65 \pm 16.08 \mathrm{~min}$, whereas the meantime for obtaining blood for culture was $106.93 \pm 72.64 \mathrm{~min}$ and administration of first dose antibiotic was $134 \pm 84.04 \mathrm{~min}$ [Table 4].

Out of 75 patients, 30 patients did not achieve early treatment goals. This was because six were under triaged, seven had physicians delay in diagnosis of sepsis, eleven had logistical delay in management and six had financial constraints [Table 5].

\section{DISCUSSION}

Sepsis is a global health problem, causing high mortality and morbidity. ${ }^{[6,7]}$ Majority of these patients are treated initially in the emergency departments (ED). The sequelae of it result in disability which consumes a large proportion of our resources. ${ }^{[6]}$ Hence a better treatment protocol is required for the benefits of such patients. Surviving Sepsis Campaign introduced a bundle of care for treatment of sepsis patients. ${ }^{[5]}$ It includes two bundles, to be completed within 3 and 6 hours to improve the outcome of patients.

The reasons for not achieving the sepsis bundle care in this study were due to multiple factors. The factors were as follows.

\section{Under triaging}

Any individual who was up-triaged on the first contact by a physician was considered as under triaged.

The triage in this institute is a nurse-led triage system. Six individuals were re-triaged by the ED physician on first contact to a higher triage category.

Triage itself is a new concept in this country were ED is in its infancy. ${ }^{[8]}$

This delay reflects the lack of training of nurses on the concepts of triage. Implementation of a sepsis alert or screening tools for triage also might improve the management of sepsis patients. ${ }^{[9]}$

\section{Diagnostic delay from the physician's side}

In the event a goal was not met and the delay was mostly contributed by the physician not seeing the patient, or if the delay was due to the physician not labeling the term

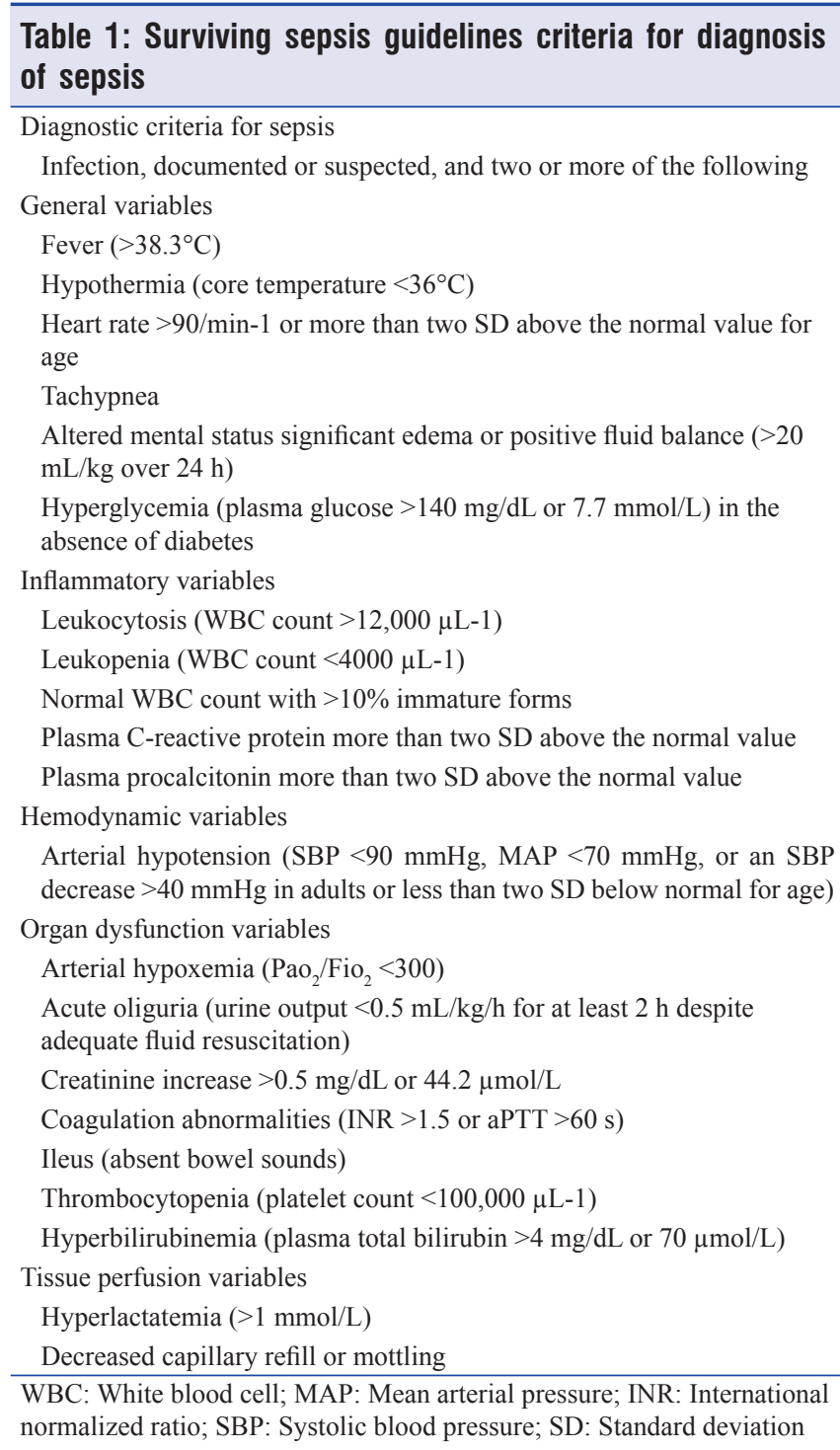

Table 2: 3-h bundle achievement

\begin{tabular}{lc}
\hline & $\begin{array}{c}\mathbf{3} \text { h bundle } \\
\text { Category (\%) }\end{array}$ \\
\hline Achieved & $53(70.7)$ \\
Not Achieved & $22(29.3)$ \\
Total & $75(100.0)$ \\
\hline
\end{tabular}

Table 3: 6-h bundle achievement

\begin{tabular}{lc}
\hline & $\begin{array}{c}\mathbf{6} \text { h bundle } \\
\text { Category (\%) }\end{array}$ \\
\hline Achieved & $63(84.0)$ \\
Not achieved & $12(16.0)$ \\
Total & $75(100.0)$ \\
\hline
\end{tabular}

"sepsis" on initial contact but waiting to get confirmation from their supervisor for lack of their experience of treating the same, it was marked as diagnostic delay by the independent observer. 
Kassyap, et al.: Factors affecting early treatment goals of sepsis patients

\begin{tabular}{lcccc}
\hline Table 4: Mean time taken to achieve 3 -h bundle components & & \\
\hline & $\boldsymbol{n}$ & Minimum (min) & Maximum (min) & Mean \pm SD \\
\hline Lactate time & 75 & 5.00 & 105.00 & $20.6533 \pm 16.08633$ \\
Blood culture time & 75 & 10.00 & 270.00 & $106.9333 \pm 72.64265$ \\
First dose antibiotic time & 75 & 30.00 & 345.00 & $133.8000 \pm 84.04005$ \\
\hline
\end{tabular}

SD: Standard deviation

\begin{tabular}{lc}
\hline $\begin{array}{l}\text { Table 5: Distribution of delay factors and achievement of } \\
\text { early treatment goals }\end{array}$ \\
\hline Factors & $\boldsymbol{n}(\%)$ \\
\hline Under triage & $6(8.00)$ \\
Physicians delay & $7(9.33)$ \\
Logistical delay & $11(14.67)$ \\
Financial constraints & $6(8.00)$ \\
Patients achieved bundles & $45(60.00)$ \\
Total & $75(100)$ \\
\hline
\end{tabular}

Even for practitioners with formal medical education, lack of exposure to acute care medicine during training and limited opportunities for continuing medical education suggest that many have insufficient knowledge of best practices for sepsis diagnosis and treatment. ${ }^{[9]}$ In India, EM is yet to be included under the undergraduate curriculum. ${ }^{[8]}$

In a busy ED with resource constraints, it is often the junior most doctor that first approaches the patient. Diagnosing "sepsis" means employment of more resources.

Whether physicians in a resource-constrained environment are apprehensive in diagnosing sepsis, since the term itself warrants the employment of additional resources or if their sensitization to sepsis is lower in a low-income country could be contributing factors that may be further studied.

\section{Logistical delay}

Any delay in admission procedures, technical issue with hospital information system, patient transfer, waiting period in radiology, and laboratory was noted as a logistical delay.

This reflects the lack of sensitization among the medical fraternity with regards to sepsis. Lack of any sepsis alert concept which is time focused is reflected in this delay. Reliable point of care tests in the ED may help in early diagnosis and may reduce logistical delays.

\section{Financial constraints}

In eleven individuals, the delay in achieving the goal was attributed to the financial constraints of the patient or their caregiver. This number probably in the authors' opinion is a gross under-representation of the actual impact finance has on the care of these patients.

It is to be noted that only patients who were admitted to the institute were included in the study. The institute in which this study was conducted as a mission hospital that charged Indian Rupees 180/-(approximately 3 USD) as admission fees during the period of the study was conducted. Although no emergency care was denied to them, these individuals were not included since they did not get admitted.

In achieving the 6-h goals, the need for continuous monitoring both invasive and noninvasive would warrant more resources. People often will not be able to afford the care since most have to pay out of their own pockets. Time spent discussing with patients relative or bystanders regarding the anticipated financial burden is something unique in resource-constrained situations which would invariably lead to decision-making delays.

\section{Limitations of the study}

This model lacks external validation because this was an observational study conducted at a single tertiary center with a limited sample size of 75 patients. The treating physicians were not blinded to the conduct of the study, making performance bias an anticipated issue. Although it was an independent observer that quantified the reason for the delay, the study was subjective in the assessment of the same.

\section{Conclusion}

The guideline prescribed 3-h bundle was achieved in 70.7\% of cases whereas 6 -h bundle was achieved in $84 \%$ of cases in this institute. The sepsis bundle care was not achieved because of various factors such as under triaging, delay in diagnosis, logistical delay, and financial constraints.

The factors delaying management of septic patients are a myriad in resource-constrained hospital. Specific to institutes with limited resources, there is a need for sensitization of medical fraternity nurses and doctors alike about the management of sepsis, implementation of sepsis treatment protocols, insurance policies, and point of care diagnostics.

\section{Acknowledgment}

The authors would like to acknowledge Mrs. Liji Thomas for the statistical work done for the paper.

\section{Financial support and sponsorship \\ Nil.}

\section{Conflicts of interest}

There are no conflicts of interest.

\section{RefEREnCES}

1. Martin GS, Mannino DM, Eaton S, Moss M. The epidemiology of sepsis in the United States from 1979 through 2000. N Engl J Med 2003;348:1546-54.

2. Póvoa P, Coelho L, Almeida E, Fernandes A, Mealha R, Moreira P, 
et al. C-reactive protein as a marker of ventilator-associated pneumonia resolution: A pilot study. Eur Respir J 2005;25:804-12.

3. Chatterjee S, Bhattacharya M, Todi SK. Epidemiology of adult-population sepsis in India: A single center 5 year experience. Indian J Crit Care Med 2017;21:573-7.

4. Koscove EM. Sepsis and septic shock. Infectious Disease in Emergency Medicine. Philadelphia: Lippincott-Raven Publishers; 1998. p. $129-52$.

5. Dellinger RP, Levy MM, Rhodes A, Annane D, Gerlach H, Opal SM, et al. Surviving sepsis campaign: International guidelines for management of severe sepsis and septic shock, 2012. Intensive Care Med 2013;39:165-228.

6. Jawad I, Lukšić I, Rafnsson SB. Assessing available information on the burden of sepsis: Global estimates of incidence, prevalence and mortality. J Glob Health 2012;2:010404.

7. Stevenson EK, Rubenstein AR, Radin GT, Wiener RS, Walkey AJ. Two decades of mortality trends among patients with severe sepsis: A comparative meta-analysis. Crit Care Med 2014;42:625-31.

8. Aggarwal P, Galwankar S, Kalra OP, Bhalla A, Bhoi S, Sundarakumar S, et al. The 2014 academic college of emergency experts in India's education development committee (EDC) white paper on establishing an academic department of emergency medicine in India - Guidelines for staffing, infrastructure, resources, curriculum and training. J Emerg Trauma Shock 2014;7:196-208.

9. Papali A, McCurdy MT, Calvello EJ. A "three delays" model for severe sepsis in resource-limited countries. J Crit Care 2015;30:861.e9-14. 